\title{
Regular Threshold-Energy Increase with Charge for Neutral-Particle Emission in Collisions of Electrons with Oligonucleotide Anions
}

\author{
T. Tanabe, ${ }^{1}$ K. Noda,${ }^{2}$ M. Saito, ${ }^{3}$ E. B. Starikov, ${ }^{4}$ and M. Tateno ${ }^{5}$ \\ ${ }^{1}$ High Energy Accelerator Research Organization (KEK), Tsukuba 305-0801, Japan \\ ${ }^{2}$ National Institute of Radiological Sciences, Anagawa, Chiba 263-8555, Japan \\ ${ }^{3}$ Kyoto Prefectural University, Kyoto 606-8522, Japan \\ ${ }^{4}$ Karolinska Institute, CSB NOVUM, S-14157 Huddinge, Sweden \\ ${ }^{5}$ National Institute of Advanced Industrial Science and Technology, Tsukuba 305-8568, Japan
}

(Received 9 July 2003; published 23 July 2004)

\begin{abstract}
Electron-DNA anion collisions were studied using an electrostatic storage ring with a merging electron-beam technique. The rate of neutral particles emitted in collisions started to increase from definite threshold energies, which increased regularly with ion charges in steps of about $10 \mathrm{eV}$. These threshold energies were almost independent of the length and sequence of DNA, but depended strongly on the ion charges. Neutral particles came from breaks of DNAs, rather than electron detachment. The step of the threshold energy increase approximately agreed with the plasmon excitation energy. It is deduced that plasmon excitation is closely related to the reaction mechanism.
\end{abstract}

PACS numbers: $34.80 . \mathrm{Ht}, 87.90 .+\mathrm{y}$

The interactions between DNA and photons or electrons are attracting much interest, related to our understanding of the elementary process, radiation damage, and radiation therapy. In photon-DNA collisions, a threshold of single-strand breaks was observed around $10 \mathrm{eV}$ [1]. In electron-DNA collisions, single- and doublestrand breaks have been observed around 10 and $20 \mathrm{eV}$, which are deduced to be caused by rapid decays of transient molecular resonances localized on the DNA's basic components in the gas phase, or in homogeneous films [2]. On the other hand, it was shown that electrons at energies below the threshold for electron excitation $(<3 \mathrm{eV})$ effectively decompose gas-phase uracil, generating a mobile hydrogen radical and the corresponding closed-shell uracil fragment anions [3]. There have also been many experiments on the collision induced dissociation (CID) of multiply charged DNA anions in gas targets [4]. In contrast to studies on electron-neutral DNA collisions and CID on DNA anions, little has been reported on electron-DNA anion collisions, probably due to a lack of experimental means. Moreover, the idea of the collective motion of electrons in the form of plasmon oscillations was not used to interpret the above-cited experiments, although the bulk plasmon concept has been long known to be relevant to DNA high-energy physics [5-8] and DNA surface plasmons were studied and used widely for applications (cf., e.g., [9]).

Recent progress in electrostatic storage rings has allowed the storage of biomolecular ions with long lifetimes and moderate beam intensities [10,11]. Furthermore, by adding an electron target of the merging beam type $[12,13]$, a new way of studying electronbiomolecular ion collisions in the gas phase was opened. In this Letter, we consider collisions between electrons and $m$-mer oligonucleotide anions $(m: 2-14)$ with charge states of $n(n: 1-4)$.
Our experimental apparatus and procedures have been described previously [14]. Synthesized DNA oligomers were from Nippon EGT Co. Japan. The samples were dissolved in a water $(50 \%)$ and methanol $(50 \%)$ mixture with a biomolecule concentration of about $0.1 \mathrm{mM}$, and sprayed in an electronspray ion source (ESI). Deprotonated oligonucleotide anions emitted from the ESI were first stored in a linear ion trap for about $10 \mathrm{~s}$, in order to increase the ion-beam intensities. Ions were then ejected as a bunch, and conveyed to an accelerator tube, where they were accelerated to $20 \mathrm{keV} /$ charge. After being mass analyzed with a mass-resolving power of about $10^{3}$, ions were injected into an electrostatic storage ring [15] with a circumference of $8.1 \mathrm{~m}$, as shown in Fig. 1. The lifetimes of ions were about $15 \mathrm{~s}$ for singly charged anions and about $5 \mathrm{~s}$ for anions with a charge of 4 under a vacuum of $4 \times$ $10^{-11}$ Torr. An electron beam with a current of $13 \mu \mathrm{A}$ and a diameter of $20 \mathrm{~mm}$ guided in a solenoid field of $30 \mathrm{G}$ merged with an ion beam with a diameter of about $6 \mathrm{~mm}$ in a $20-\mathrm{cm}$ long interaction region. The energy resolution was about $0.2 \mathrm{eV}$ at an electron energy of $1 \mathrm{eV}$, which was dominated by a space-charge depression, although the longitudinal and transverse electron temperatures were lower due to an adiabatic expansion of the electron beam by a factor of 30 . The space-charge depression decreased with an increase of the electron energy. The electron energies were variable from 1 to $100 \mathrm{eV}$. The actual electron energy $\left(E_{e}\right)$ at an acceleration voltage of $V_{a}$ is given by the equation $E_{e}=V_{a}+V_{0}-K I_{e} / \sqrt{E_{e}}$, where $I_{e}$ is the electron current, and $V_{0}$ and $K$ are constants at a fixed electron current. These constants were determined by measuring the rates of neutrals emitted from dissociative recombinations of $\mathrm{H}_{2}^{+}, \mathrm{D}_{2}^{+}$, and $\mathrm{D}_{3}^{+}$at $20 \mathrm{keV}$ as a function of the electron energies, where the electron recombination rates have maxima at zero relative 


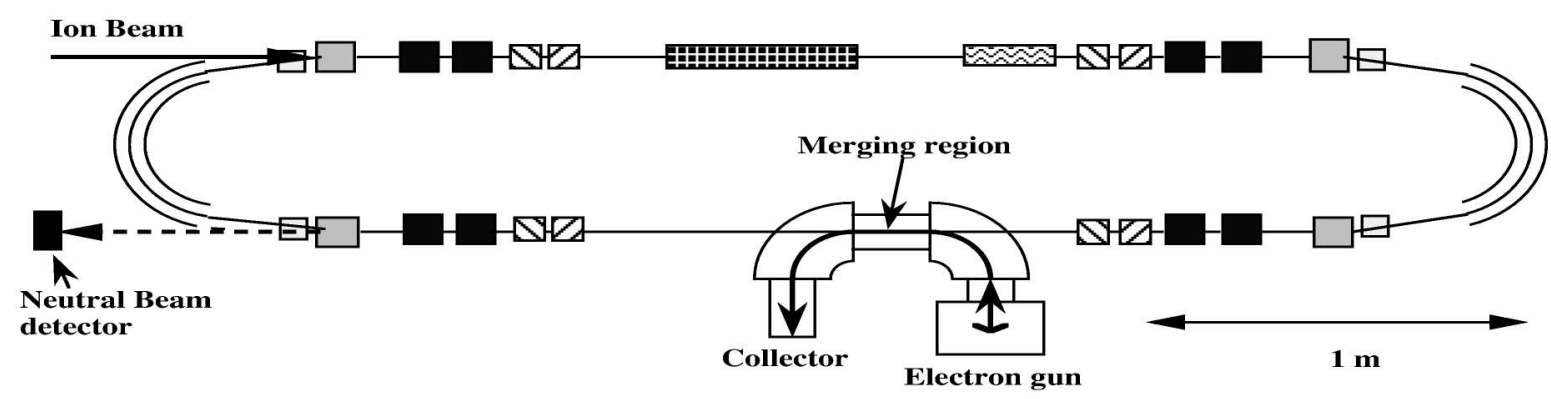

FIG. 1. Experimental setup for an electrostatic storage ring with a merging electron beam device and a neutral beam detector.

energies between the ions and the electrons, corresponding to electron energies of $5.45,2.72$, and $1.82 \mathrm{eV}$, respectively.

The neutral products were detected by a microchannel plate with a phosphor anode having a diameter of $77 \mathrm{~mm}$ installed in a vacuum extension $3 \mathrm{~m}$ downstream from the merging section (see Fig. 1). In order to deexcite vibrationally excited ions, they were first stored in the ring for a time of $0.5 \mathrm{~s}$ after injection, and then measurements were started. In order to discriminate ion-electron collisions from ion-residual gas collisions, the electron beam was chopped at a time width of $0.25 \mathrm{~s}$. As the ion beam intensities decreased with time, ions were dumped and refilled in the ring every $10.5 \mathrm{~s}$. The rate coefficient at an ion velocity of $v_{i}$ and an electron density of $\rho_{e}$ is given by

$$
\left\langle v_{r} \sigma\right\rangle=\frac{1}{\varepsilon} \frac{N-N_{B G}}{N_{i}} \frac{v_{i}}{\rho_{e} L},
$$

where $v_{r}$ is the relative velocity, $N_{i}$ is the number of ions, $L$ is the interaction length, and $\varepsilon$ is the detection efficiency. $N$ and $N_{B G}$ are the number of neutrals with and without an electron beam, respectively. The relative rate coefficients were extracted assuming $N_{i} \propto N_{B G}$ and neglecting the efficiency.

The results of the measurements are shown in Fig. 2 on 2-14 mer DNAs with charge states of 1-4. The length and sequence of DNAs were chosen arbitrarily. In Fig. 2, with an increase in the charge state, longer DNAs were adopted, because charge states at a given DNA distribute over a range, and the charge state with the highest ion intensities shifts to higher values with an increase in the length of DNA. In order to study the dependence of the threshold energies on the length and sequence of DNA, measurements were performed on DNAs with the same charge, but having a different length and sequence. As can be seen in the figure, the threshold energies are almost independent of the length and the sequence, but depend strongly on the charge state. They increase regularly with charge at an interval of about $10 \mathrm{eV}$, although the thresholds become obscure with an increase in the charge state.

There are two possibilities for neutral particle production: One is electron detachment, resulting in neutraliza- tion; the other is cleavage of molecules, including strand breaks, resulting in the emission of neutral fragments. In order to study the probabilities of these processes qualitatively, we measured the number of neutrals arriving

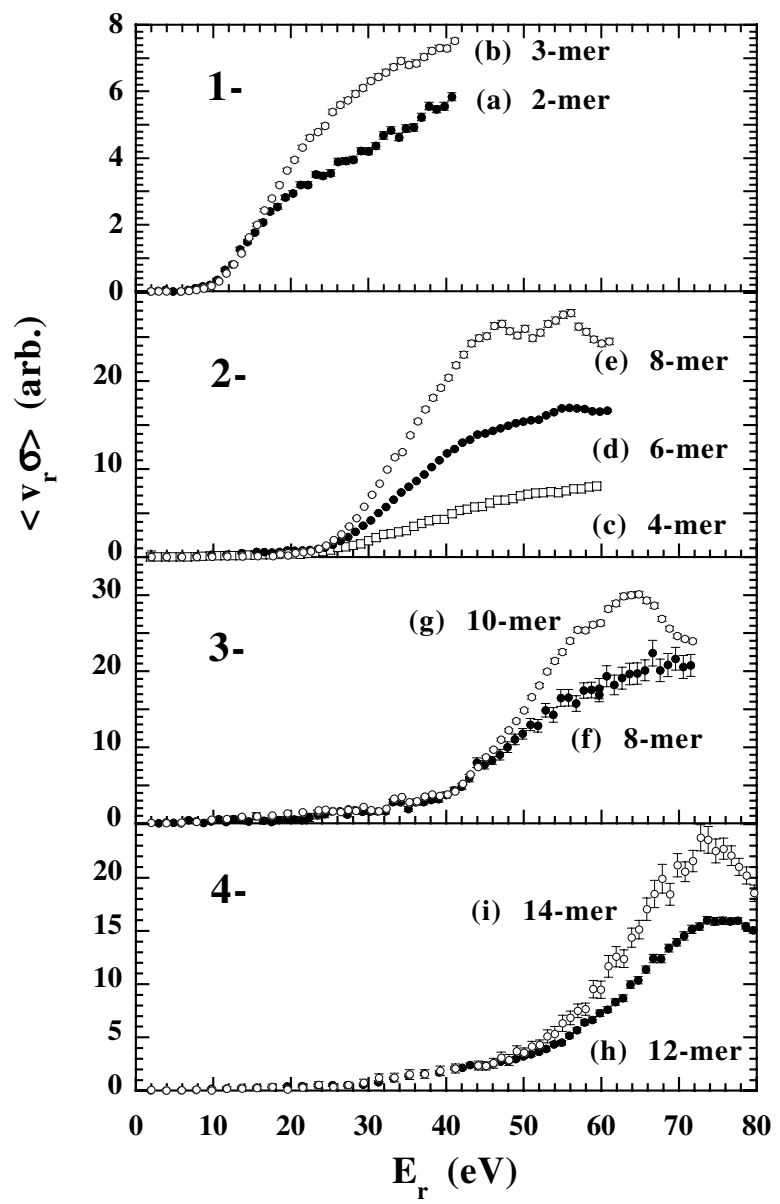

FIG. 2. Neutral particle production rate as a function of the relative energies $\left(E_{r}\right)$ in collisions between electrons and deprotonated DNA anions. $E_{r}$ is given by $\sqrt{E_{r}}=\sqrt{E_{e}}-$ $\sqrt{\left(m_{e} / m_{i}\right) E_{i}}$, where $E_{i}$ is the ion energy, and $m_{e}$ and $m_{i}$ are electron and ion masses, respectively. The charge states and lengths are given in the figure. The sequences are (a) TG, (b) ATG, (c) ATGC, (d) ATGCTG, (e),(f) ATGCATGC, (g) ATGCATGCTG, (h) ATGCATGCATGC, and (i) ATGCATGCATGCAT. 
simultaneously at the detector with a two-dimensional imaging technique [16] using a charge-coupled device camera. Measurements were performed with a position resolution of about $0.2 \mathrm{~mm}$ and a time window of about $20 \mu \mathrm{s}$. The number of neutrals was measured for a singly charged 2-mer [sequence: d(TG)] anion, with electron beams having relative energies of 4 and $35 \mathrm{eV}$ which are below and above the threshold, respectively (see Fig. 2). The average number of neutrals simultaneously arriving at the detector clearly increases at $35 \mathrm{eV}$ compared with at $4 \mathrm{eV}$, although quantitative estimation is hard due to the lack of information on the detection efficiency of the microchannel plate and also due to the difficulty in removing the contribution from residual gas from the two-dimensional imaging data. This means that neutrals emitted at energies higher than the threshold energies come mainly from breaks of oligonucleotides, rather than electron detachment.

Earlier, it was speculated [6-8] that, when DNA is subject to direct hits by high-energy particles, collective electron excitations in the form of bulk plasmons could act as the first stage of the DNA response, triggering a succession of events, leading at last to DNA strand breaks. To support this idea, some work [6-8] adopted the conventional solid-state viewpoint, and took into account only all of the valence electrons of the DNA bases. However, in our experimental setup, high-energy electrons hit separate DNA oligomer anions. Hence, we consider instead that all of the valence electrons of a separate finite oligonucleotide anion (bases and phosphate backbone) are involved in the response to electron hits by exhibiting plasmonlike oscillations. In order to study the possibility of bulk plasmon excitation, we calculated the energy needed to excite one plasmon, given by

$$
\hbar \omega_{p}=\hbar \sqrt{\frac{4 \pi n_{V} e^{2}}{m}},
$$

where $n_{V}$ is the electron density, and $e$ and $m$ are the electron charge and mass, respectively. The $n_{V}$ is given by the number of valence electrons divided by the volume of the oligonucleotide, which was calculated based on the Voronoi procedure implemented in a computer program of PROVE by Pontius et al. [17] and slightly modified by us. The results for 3-, 6-, 8-, and 12-mer oligonucleotides [sequence: d(ATG), d(ATGCTG), d(ATGCATGC), and d(ATGCATGCATGC)] corresponding to the data in Fig. 2 are 9.2, 10.0, 10.3, and $10.5 \mathrm{eV}$, respectively. Thus, the calculated plasmon energies gradually increase with the length, and saturate. Moreover, the calculated plasmon energies are also slightly dependent on the sequence. For example, the energy for 6-mer DNA with a sequence of d(GGGGGG) is $10.2 \mathrm{eV}$. Interestingly, the experimental threshold energies approximately agree with the corresponding plasmon energies multiplied by an integer number equal to the value of the corresponding oligo- nucleotide ion charge. In other words, high-energy electron hits seem to simultaneously excite a larger number of plasmon quanta, the higher being the DNA oligomer anion charge. This might bear a deep biological significance in explaining the physical mechanisms of DNA resistance in regard to a singular hit by a high-energy particle. However, the detailed mechanisms of this phenomenon are still unclear.

Finally, a very recent publication [18] should be noted which deals with low-energy electron diffraction and resonances in DNA. An interesting model based upon $R$-matrix scattering theory is proposed there to explain the relevant experimental results. However, we cannot immediately apply this model to our data, since our electron energy range extends up to $100 \mathrm{eV}$.

In conclusion, this Letter reports on a marked regular threshold energy increase in electron-oligonucleotide anion collisions. Apparently, more work is required before satisfying explanations can be given for this simple phenomenon. We hope that these results will help to stimulate further research both experimentally and theoretically.

The authors thank H. Takagi for discussions related to atomic processes, K. Chida and M. Yoshizawa for their helpful cooperation on constructing the vacuum system, and Y. Itoh for preparing biomolecule solutions. The present calculations for obtaining the volume of DNA molecules were carried out by using the SGI Origin in the Computer Center for AFFR, MAFF Japan.

[1] K. Hieda, K. Suzuki, T. Hirono, M. Suzuki, and Y. Furusawa, J. Radiat. Res. 35, 104 (1994).

[2] B. Boudaïffa, P. Cloutier, D. Hunting, M. A. Huels, and L. Sanche, Science 287, 1658 (2000).

[3] G. Hanel, B. Gstir, S. Denifl, P. Scheier, M. Probst, B. Farizon, M. Farizon, E. Illenberger, and T. D. Märk, Phys. Rev. Lett. 90, 188104 (2003).

[4] S. Schürch, E. Bernal-Méndez, and C. J. Leumann, J. Am. Soc. Mass Spectrom. 13, 936 (2002).

[5] C. D. Johnson and T. B. Rymer, Nature (London) 213, 1045 (1967).

[6] J. Jäger and J. Ladik, Phys. Lett. 28A, 328 (1968).

[7] J. Ladik, J. Mol. Struct. (THEOCHEM) 277, 109 (1992).

[8] J. Ladik, H. Fruechtl. P. Otto, and J. Jäger, J. Mol. Struct. 297, 215 (1993).

[9] R. J. Heaton, A.W. Peterson, and R. M. Georgiadis, Proc. Natl. Acad. Sci. U.S.A. 98, 3701 (2001).

[10] T. Tanabe and K. Noda, Nucl. Instrum. Methods Phys. Res., Sect. A 496, 233 (2003).

[11] J. U. Andersen, P. Hvelplund, S. B. Nielsen, S. Tomita, S. P. Møller, U.V. Pedersen, J.S. Forster, and T. J. D. Jørgensen, Rev. Sci. Instrum. 73, 1284 (2002).

[12] T. Tanabe, K. Noda, and I. Watanabe, Workshop on Ion Beam Cooling-Toward the Crystalline Beam, Kyoto, 2001 (World Scientific, Singapore, 2002), p. 198. 
[13] T. Tanabe, S. Lee, K. Noda, I. Watanabe, and E. Syresin, Proceedings of the 8th European Particle Accelerator Conference, Paris, 2002 (CERN, Geneva, 2002),p. 632.

[14] T. Tanabe, K. Noda, M. Saito, S. Lee, Y. Ito, and H. Takagi, Phys. Rev. Lett. 90, 193201 (2003).

[15] T. Tanabe, K. Chida, K. Noda, and I. Watanabe, Nucl. Instrum. Methods Phys. Res., Sect. A 482, 595 (2002).
[16] M. Saito, Y. Haruyma, T. Tanabe, I. Katayama, K. Chida, T. Watanabe, Y. Arakaki, I. Nomura, T. Honma, K. Noda, and K. Hosono, Phys. Rev. A 61, 062707 (2000).

[17] J. Pontius, J. Richelle, and S. J. Wodak, J. Mol. Biol. 264, 121 (1996).

[18] L. G. Caron and L. Sanche, Phys. Rev. Lett. 91, 113201 (2003). 